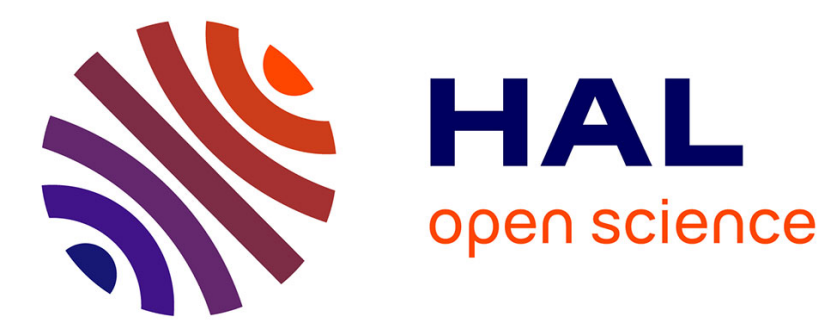

\title{
Development and characterization of an adaptable aerosolized methylene diphenyl diisocyanate generation system
}

Simon Aubin, Loïc Wingert, Sébastien Gagné, Livain Breau, Jacques Lesage

\section{- To cite this version:}

Simon Aubin, Loïc Wingert, Sébastien Gagné, Livain Breau, Jacques Lesage. Development and characterization of an adaptable aerosolized methylene diphenyl diisocyanate generation system. Environmental Science: Processes \& Impacts, In press, 10.1039/D1EM00183C . hal-03385808

\section{HAL Id: hal-03385808 \\ https://hal.science/hal-03385808}

Submitted on 19 Oct 2021

HAL is a multi-disciplinary open access archive for the deposit and dissemination of scientific research documents, whether they are published or not. The documents may come from teaching and research institutions in France or abroad, or from public or private research centers.
L'archive ouverte pluridisciplinaire HAL, est destinée au dépôt et à la diffusion de documents scientifiques de niveau recherche, publiés ou non, émanant des établissements d'enseignement et de recherche français ou étrangers, des laboratoires publics ou privés. 


\title{
Development and Characterization of an Adaptable Aerosolized Methylene Diphenyl Diisocyanate Generation System
}

\author{
Simon Aubin ${ }^{1,2}$, Loïc Wingert ${ }^{1}$, Sébastien Gagné ${ }^{1}$, Livain Breau ${ }^{2}$, Jacques Lesage ${ }^{2}$ \\ ${ }^{1}$ Institut de recherche Robert-Sauvé en santé et en sécurité du travail (IRSST), Qc, Canada \\ ${ }^{2}$ Université du Québec à Montréal (UQAM), Chemistry Department, Qc, Canada
}

\section{Abstract}

Reactive semivolatile contaminants, such as isocyanates, can be particularly difficult to assess in occupational environments. While standard methods exist for isocyanates, there are still significant differences between the results they provide for various occupational environments or processes. This study presents the validation of a laboratory system for the generation of controlled atmospheres of isocyanates. A system consisting of different modules generated airborne methylene diphenyl diisocyanate (MDI) by nebulizing a solution into mixing and exposure chambers with control of flow rate, temperature, and relative humidity. Sampling was performed through an eight-port flow splitter that allowed only very slight within-test variability. MDI was measured using the Asset EZ4-NCO ${ }^{\circledR}$ and a modified version of the Iso$\mathrm{Chek}^{\circledR}$ sampling system. MDI specific particle-size distribution was measured by a Marple Sierra cascade. Aerosol real-time monitoring was performed using a condensation particle counter, an electrical low-pressure impactor (ELPI+), and an optical aerosol monitor, providing additional information on system stability and particle-size distribution of the generated aerosol. The system was able to generate MDI concentration levels ranging from 4 to $233 \mu \mathrm{g} / \mathrm{m}^{3}$, with a steady-state level reached within 5 minutes, and with well-documented intra-test and inter-test variability (RSD of $4 \%$ and $15 \%$, respectively). Accuracy and representativeness of MDI data were confirmed by the agreement between MAMA and Asset EZ4-NCO (used as reference), with a mean bias of 3\%. Using the Asset EZ4-NCO capability, the vapor-particle partitioning of MDI was evaluated to be $8 \%$ and $92 \%$, respectively, at a concentration ranging from 20 to $25 \mu \mathrm{g} / \mathrm{m}^{3}$. The system may therefore be used for exhaustive method intercomparison studies and could also be adapted to generate other emission types of semivolatile compounds.

\section{Environmental Significance Statement}

Studies have been conducted in recent years to gain a better understanding of the behavior of semivolatile workplace aerosols in air-sampling devices. Little attention has been paid, however, to reactive semivolatile aerosols, such as isocyanates, which require chemical stabilization within the air-sampling device. The goal of this article is to develop and characterize a userfriendly test bench dedicated to comparing sampling methods while allowing the main environmental parameters to be controlled for. A system like this is a tool that will ultimately provide a better understanding of the differences observed in existing measurement methods for reactive semivolatile aerosols like isocyanates. 


\section{Introduction}

The measurement of airborne chemical contaminants in the workplace can be performed by a variety of methods, including direct reading instruments ("on site"), or by integrated sampling on a sampling device followed by laboratory analysis. The latter method is still the approach used today for the evaluation of two-phase airborne substances, i.e., in vapor and particulate phases. These substances are called "semivolatile," although the categorization is not entirely standardized.(1-3) This category includes air contaminants commonly found in workplaces, such as metalworking fluids (or oil mists), plasticizers (e.g. phthalates) as well as biocidal products can also be considered to be "semivolatile". PAHs and certain pesticides are examples for which measurement methods have been developed over the years.(4-6) These methods generally apply the principles of filtration and adsorption to sample the particulate and vapor phases respectively. Some semivolatile compounds have chemical properties that make it necessary to stabilize them as soon as they are collected in the sampling device. Chemisorption, a subcategory of absorption, must therefore be exploited via a liquid (impinger) or solid support (impregnated filter) containing a chemical reagent that stabilizes highly reactive compounds (a process called "derivatization") before they are released or that reacts with other chemical species thus making their analysis impossible. Failure to follow such a sampling approach would lead to incorrect measurement of the concentration of the compounds in the air. The potentially complex and evolving two-phase nature of such highly reactive compounds therefore requires that the sampling device be adapted so as to be effective for a variety of atmospheric applications with varying vapor-vs.-particle proportions and particle-size distribution. Achieving ideal efficiency for such a variety of scenarios is still a challenge today in a context where, for example, the recommendations of the American Conference of Governmental Industrial Hygienists $\left(A C G I H^{\circledR}\right)$ increasingly take into account the semivolatile nature of contaminants by assigning them the notation "inhalable fraction and vapor" (IFV) without necessarily suggesting a standardized method for sampling and analysis. $(7,8)$

Isocyanate substances are a group particularly affected by the challenges described above. They are notorious irritants and sensitizers and are recognized as a causative agent of occupational asthma,(9-14) leading to the establishment of occupational exposure limits of the order of a few parts per billion $(\mathrm{ppb}) .(8,15)$ In addition, their use in the workplace is widespread, given that they are a raw material in the manufacture of polyurethane products (paint, varnish, insulating foam, mattresses, glue, adhesives, etc.).(16) These different manufacturing processes involve types of isocyanate emissions into the air with very different characteristics, such as spraying, heating, or stirring. Another issue is the rate of polymerization of the isocyanates, which can be relatively slow or very fast depending on the presence or absence of a copolymer ${ }^{a}$ within the aerosols emitted by the process.(17)

A number of methods, with their specific reagents, have been developed over the last few decades, with the first generation using an impinger (for absorption in a liquid) to ensure ideal derivatization.(18-21) The transposition of chemisorption to a solid support, for userfriendliness, led to the development of impregnated filter methods.(18-21) This transposition highlighted the limitations of the solid support, where the access of isocyanate to the derivation

${ }^{a}$ Copolymer: polyfunctional alcohols or amines involved in a polymerization reaction with isocyanate functions. 
reagents would seem to explain the underestimation of this type of method, compared to the impinger for certain types of emissions.(17, 22, 23) In fact, an excessively large accumulation of particles on an impregnated filter can block access to the reagent, as can the collection of large, rapidly reacting particles $(>10 \mu \mathrm{m}$ ) which would only be partially in contact with the filter reagent at the time of their collection. However, to our knowledge, these phenomena have never been clearly demonstrated experimentally. The accumulation phenomenon has been indirectly demonstrated by means of a comparative study with and without field desorption.(24) Moreover, although it is a rational explanation, the phenomenon of large, fast-reacting particles has only been summarily characterized by electron microscope observations.(25)

More recently, methods based on different collection principles-"denudation"(26) or centrifugation(27)-have been developed. Our research team carried out method comparison studies in the workplace by focusing on processes that generate various typical isocyanate atmospheres. $(28,29)$ These studies revealed trends in inter-method biases that seemed specific to the types of isocyanate emissions. However, the studies could not explain, with supporting evidence, certain biases observed between reference methods for which significantly similar measurements were expected.

Understanding the mechanisms of collection and access to the reagent from a collection medium requires controlling a majority of the parameters. However, by their nature, field studies are not very conducive to achieving this goal. Therefore, the use of a laboratory test bench is preferred. Systems for the generation of isocyanates have been designed in the past for the purposes of method development or comparison.(30-33) While meeting the intrinsic needs of the studies cited, their use was complex and not always compatible with different modes of generation.

This article presents the development of a system capable of generating controlled atmospheres of isocyanates in the laboratory for the purpose of studying different systems for isocyanate sampling. This type of development requires the implementation of simple-to-complex generation strategies and elements of control and characterization. The study objective was to develop, validate, and characterize a simple modular system for the generation of isocyanates. Methylene diphenyl diisocyanate (MDI) was the compound selected for the development of the system, based on its physical properties and its use throughout the polyurethane product industry. The work presented here characterizes the performance of the system in terms of concentration range, intra- and inter-test variability, temporal and spatial stability, particle-size distribution, and assessment of the vapor and particulate phase partition. The use of real-time particle measurement instruments complements MDI's chemical analyses (typically performed by liquid chromatography coupled to ultraviolet or mass spectrometry detection) for a comprehensive understanding of the atmospheres produced by the system.

This proof of concept is part of an increasingly complex process that will serve as the basis for the subsequent addition of modules which, over time, will allow the production of controlled atmospheres representative of real industrial processes. This breakthrough will thus make it possible to study the performance of different methods for measuring airborne chemical contaminants, in particular isocyanates, in a controlled laboratory setting while providing a significant gain in representativeness. 


\section{Materials and Methods}

\section{Part 1 - Generation System}

The generation system, set up in a walk-in fume hood, consisted of different modules, which are shown in Figure 1. Airborne MDI was generated by nebulization of a solution of MDI (SigmaAldrich, USA) in hexane (Fisher Scientific, Canada) at concentrations ranging from 20 to $200 \mathrm{mg} / \mathrm{L}$, depending on the desired concentration. The nebulizer was a Meinhard TQ-30-A3 (Meinhard, USA) for analysis by inductively coupled plasma. This type of nebulizer principle transfers completely the aspirated solution into the system and therefore provides a stable feed of $\mathrm{MDI}$ since no solvent evaporation occurs in the MDI solution container. An overflow container was connected downstream of the nebulizer to collect the excess nebulized liquid. The nebulizer operated at a flow rate of $0.7 \mathrm{~L} / \mathrm{min}$ of air previously filtered by a HEPA filter from a compressor (30 psi). A mass flow controller (Brooks Instrument SLA5850S, USA) was used to control the nebulizer flow. This configuration provided the ability of generating controlled MDI concentration as long as there is solution in the container to which the nebulizer intake is connected. An environmental control module (Assay Tech MNR HCS-501, USA) routed the dilution air directly into the mixing chamber at a controlled flow rate of $20 \mathrm{~L} / \mathrm{min}$, a temperature of $22.5^{\circ} \mathrm{C}$, and $30 \%$ relative humidity. The mixing chamber (in-house laboratory made) consisted of a glass cylinder (diameter $24 \mathrm{~cm}$ and height $31 \mathrm{~cm}$ ) fitted with a tight metal cover with various openings (inlets and outlets). A computer fan ensured that the atmosphere in the chamber was homogenized before being transferred to the exposure chamber. The exposure chamber (inhouse laboratory made) was a glass cylinder (diameter $12 \mathrm{~cm}$ and height $24 \mathrm{~cm}$ ) with a cover similar to that of the mixing chamber. The air samples were taken using a flow splitter (in-house laboratory made) providing eight identical samples from a single intake in the exposure chamber. The flow splitter consisted of an aluminum cylinder in which the inlet ( $1 / 2$ " NPT) was split into eight different paths ( $1 / 4$ " NPT), each of them radially and evenly deviating by $15^{\circ}$ along the inlet axis. Preliminary tests using $\mathrm{NaCl}$ particles confirmed that each of the eight sampling ports of the flow splitter ensured a within-test variability of less than $5 \%$ (relative standard deviation, RSD) (see electronic supplementary information [ESI]). The transfer lines (inside diameter $1.3 \mathrm{~cm}$ ) from one module to the next and the Swagelok-type connections were made of stainless steel, except for the flexible tube joining the two chambers which was made of nylon (SMC, Quebec, Canada). The total flow rate and internal pressure of the system were controlled by a vacuum connected downstream of the exposure chamber, where a manual valve was used to achieve the desired air flow equilibrium, according to the air flow rate taken for the samples, and also to prevent leaks of toxic gases that could be dangerous to nearby operators. 


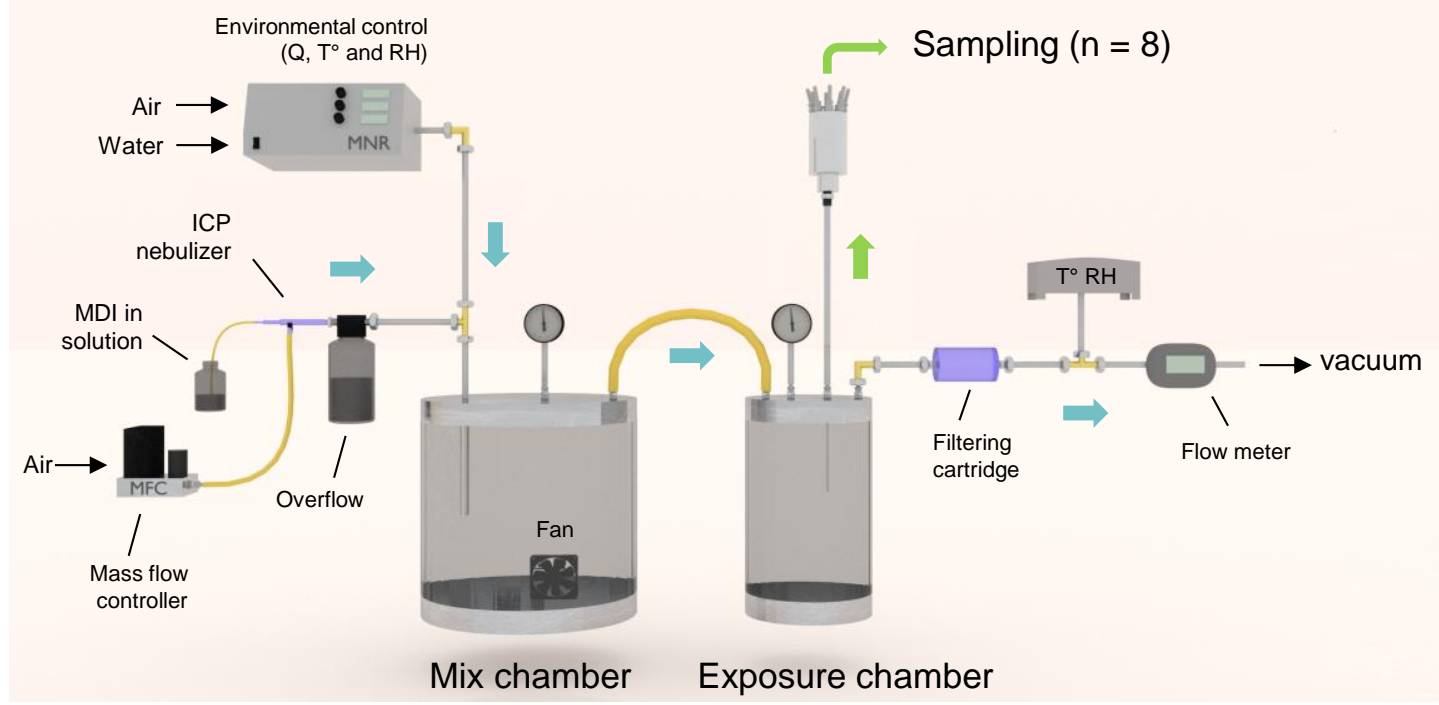

Figure 1. Modular generation system.

The internal pressure of the chambers was monitored continuously by a Type DPS pressure sensor (FSM AG, Germany). System temperature and humidity were monitored by a Vaisala probe, model HMD70Y (Finland). Total system flow was monitored in real time by a TSI 4043 mass flowmeter (USA). Both probe and flowmeter were located downstream of the exposure chamber, a HEPA filter cartridge, and activated carbon, 6704-7500 (VWR International, Ontario, Canada). Table 1 presents the environmental parameters of the generation system

Table 1. Environmental parameter values for a typical 60-min. generation test.

\begin{tabular}{cc} 
Parameters & Values and RSD \\
\hline Temperature & $22.9^{\circ} \mathrm{C}(0.8)$ \\
Relative humidity & $22.5 \%(1)$ \\
System flow rate* & $21 \mathrm{~L} / \mathrm{min}(0.5)$ \\
Residence time & \\
Mixing chamber & $40 \mathrm{~s}$ \\
Exposure chamber & $8 \mathrm{~s}$
\end{tabular}

*without sampling taking place 
Part 2 - Air Analysis

The MDI measurements were carried out mainly according to a modified version of the Iso-Chek method(34) in which only the impregnated filter with N-methyl-amino-methyl-9 anthracene (MAMA) was used in the sampler in order to analyze the vapor and particulate phases of MDI on the same filter. This modification was introduced to simplify laboratory handling of the numerous analyses required for the study and was made possible by the fact that only the monomeric form of MDI was to be analyzed. The sampling method used a $37 \mathrm{~mm}$ diameter glass fiber filter (EMD Millipore AP4003705, USA), heat treated at $400{ }^{\circ} \mathrm{C}$ for 4 hours. Each filter was individually impregnated with $400 \mu \mathrm{L}$ of a solution of $200 \mathrm{mg} / \mathrm{L}$ of MAMA (Sigma-Aldrich, USA) in toluene (EMD Millipore, USA) using an automatic pipette. The filters were dried under a nitrogen atmosphere for 4 to 6 hours away from light. The filters were then inserted into $37 \mathrm{~mm}$ polystyrene closed-face cassettes (SKC 225-2LF, USA) containing a plastic support pad (SKC 2252902, USA), which were then individually checked for leaks. Personal pumps (SKC 224-PCXR4, USA) were used to collect samples. The sampling flow rate was $1 \mathrm{~L} / \mathrm{min}$ and was measured by a flowmeter (TSI 4143 USA), and was measured again at the end of the sampling period to determine whether the flow rate had changed by more than $5 \%$. The cassettes were connected to the sampling ports by conductive silicone rubber tubing of minimum length. Each filter was extracted immediately after the end of the sampling in the desorption solution and was then analyzed by liquid chromatography (LC). The method had a detection limit of $0.01 \mu \mathrm{g}$ per sample or $0.3 \mu \mathrm{g} / \mathrm{m}^{3}$ for 30 minutes of sampling at $1 \mathrm{~L} / \mathrm{min}$. All the relevant details of the analysis method can be found in the electronic supplementary information (ESI) at the end of the article.

ISO 17734-1 (Asset) was used to validate the MDI measurements performed by the method described in the previous paragraph.(35) Its sampler consisted of a commercially available device, Asset EZ4-NCO (Sigma-Aldrich, USA), made of a denuder tube followed by an impregnated filter. This device configuration involves an additional component, a $\mathrm{V}$-filter, into the denuder tube to allow better collection efficiency for gas-phase isocyanate (26). Its derivative reagent was dibutylamine (DBA) and the analysis was performed by LC and mass spectrometry. The Asset sampler was connected to the splitter using conductive silicone rubber tubing. Low-flow personal pumps (SKC 222-3, USA) were used to collect samples with the Asset sampler. Flow rate, measured by a Mesalab Defender 510 (USA), was set at $0.2 \mathrm{~L} / \mathrm{min}$ prior to sampling and was measured again at the end of the sampling period to determine whether the rate had changed by more than $5 \%$. The samples were only desorbed at the time of the laboratory analysis, as described in the analytical procedure. The two components of the Asset sampler, denuder and filter, were analyzed separately to obtain MDI measurements differentiated by vapor (denuder) and particulate (filter) phases. Asset standards and samples were prepared and analyzed according to Halpenny et al.(36) The method had a detection limit of $0.005 \mu \mathrm{g}$ per sample or $0.8 \mu \mathrm{g} / \mathrm{m}^{3}$ for 30 minutes of sampling at $0.2 \mathrm{~L} / \mathrm{min}$. All the relevant details of this methodology can be found in the ESI.

The particle-size distribution of MDI was evaluated by a cascade impactor (Sierra Marple 298, Thermo Scientific, USA) in which were mounted $34 \mathrm{~mm}$ glass fiber slit membranes and an end filter (Air Sampling Devices FGUPMP03400, USA). Each slit membrane and end filter was individually impregnated with $400 \mu \mathrm{L}$ of MAMA solution in a manner identical to the procedure described above. The impactor flow rate was adjusted to $2 \mathrm{~L} / \mathrm{min}$ for sampling and was connected to the flow splitter with conductive silicone rubber tubing. The tube was connected 
to the impactor by means of an aluminum sampling head (laboratory made), with a $25 \mathrm{~mm}$ diameter inlet. The eight stages (slit membrane) and the end filter were individually extracted immediately after the end of the sampling in the desorption solution. The LC analysis was then performed identically to the procedure described above for the MAMA method.

The aerosol optical spectrometer (AOS) Fidas ${ }^{\circledR}$ Frog (Palas, Germany), which detects particles based on light-scattering principles, was used to count and size particles with a diameter of 0.150 to $80 \mu \mathrm{m}$ and served as a monitoring device (1-min average measurements) during generation tests. The AOS was used with its manufacturer calibration, which had been performed with polystyrene latex particles. With its aerodynamic diameter range of 0.01 to $10 \mu \mathrm{m}$ over 14 impactor stages, an electrical low-pressure impactor (ELPI+) (Dekati Ltd, Finland) was used to measure the total concentration of particles and was able to provide number and mass particle size-distributions (1-min average measurements). A condensation particle counter (CPC) (TSI 3007, USA) was used in parallel with the ELPI+. The CPC can measure particle sizes of 0.01 to $1 \mu \mathrm{m}$, and its results were used as the particle number concentration reference (1-min average measurements). Each impactor stage of the ELPI+ consists of an electrometer that detects electrical currents induced by the charged particles flowing into the instrument. These currents are then converted into concentration units. Since the conversion's accuracy relies on particle density, the effective density used by the ELPI+ algorithm was adjusted so that the ELPI+ measured the exact same particle number concentration as the CPC. It should be noted that this adjustment was required for particle number concentration measurement only, since it has a very limited effect on particle mass concentration.(37)

\section{Part 3 - Test Description and Data Processing}

The observed background of MDI and particles produced by the solvent was evaluated by performing tests when the system nebulized only hexane, from the same batch as the one used for the MDI solution which was nebulized for a given test. The solution of MDI in hexane was prepared on the same day as the tests were performed. The duration of the generation tests ranged from 15 to 67 minutes. Prior to the start of air sampling for the MDI, it was ensured that the system was stable in terms of environmental parameters and particle concentration.

All the MDI analyses mentioned were performed according to the MAMA method except where indicated. To establish the concentration range, 20 tests were performed by varying the concentration of the MDI solution between 10 and $200 \mathrm{mg} / \mathrm{L}$. The intra-test variability represented the average relative standard deviations from the different replicates recorded for each test (three to six). This variability was determined by calculating the RSD of the average of the replicates obtained during repeated tests where the concentration of the MDI solution was fixed, either $20 \mathrm{mg} / \mathrm{L}$ or $40 \mathrm{mg} / \mathrm{L}$. The stability of the MDI concentration in air over time was specifically assessed by means of a test in which four pairs of replicates followed one another over the sampling duration. Stability over time is expressed in terms of the relative standard deviation obtained from the average of each of the four pairs of samples.

The particle-size distributions determined by ELPI+ and the cascade impactors were normalized for inter-test comparison and transformed into a logarithmic unit (concentration / $d$ In particle diameter) so as to allow comparison of unequal-width particle-size channels. 


\title{
Results and Discussion
}

\author{
System Validation and Performance
}

The MDI background ranged from $<0.3$ to $0.6 \mu \mathrm{g} / \mathrm{m}^{3}$ (MAMA) for a sampled volume of $30 \mathrm{~L}$. Table 2 shows the characterization of the test bench in terms of measured MDI concentrations.

$\underline{\text { Table 2. MDI generation validation results }}$

\begin{tabular}{rc} 
Parameters & Results \\
\hline Concentration range $(n=20)$ & $4-233 \mu \mathrm{g} / \mathrm{m}^{3}$ \\
Intra-test variability $(\mathrm{n}=17)$ & $4 \%$ \\
Inter-test variability & \\
MDI $20 \mathrm{mg} / \mathrm{L}(\mathrm{n}=3)$ & $15 \%\left(8 \mu \mathrm{g} / \mathrm{m}^{3}\right)$ \\
MDI $40 \mathrm{mg} / \mathrm{L}(\mathrm{n}=5)$ & $15 \%\left(21 \mu \mathrm{g} / \mathrm{m}^{3}\right)$
\end{tabular}

Stability vs time

$\begin{array}{cc}\text { MDI specific* } & 2 \% \\ \text { Particles** } & 7 \%\end{array}$

*32 min generation; **15 to 67 min generation $(n=18)$, measured by AOS (mass concentration)

The tests demonstrated that the system could generate a stable MDI concentration in the range of 4 to $233 \mu \mathrm{g} / \mathrm{m}^{3}$ over periods of 15 to 60 minutes. This range corresponds to $8 \%$ to $457 \%$ of the exposure limit value of $51 \mathrm{\mu g} / \mathrm{m}^{3}$ (or $5 \mathrm{ppb)} \mathrm{(} 8 \mathrm{~h}$ time-weighted average), the most frequently cited standard in regulations around the world.(15) All the tests demonstrated that MDI steady-state concentration was obtained after less than 5 minutes of nebulization at fixed environmental conditions.

The intra-test variability of $4 \%$ is lower than common isocyanate evaluation method uncertainty, ranging from 10 to $25 \%)(34,35,38-40)$, and is therefore a fundamental advantage of the system, allowing the comparison of different measurement methods. Indeed, the lower the intra-test variability, the easier it is to detect a significant difference or correlation between methods, in particular by reducing the number of tests required to reach a reliable conclusion. Such a low intra-test variability can be explained by the use of a multi-port flow splitter. This highlights its capacity to enable sampling from a unique source, bypassing any variability related to atmosphere heterogeneity or spatial location or orientation.

The $15 \%$ inter-test variability revealed that the airborne MDI levels were easily controlled by the generation system. Indeed, the study of the performance of different measurement methods must be carried out on different concentration levels. Such an inter-test variability therefore makes possible, for given conditions, to anticipate and adjust accordingly the concentration level generated by the system, should the tests be conducted at low, mid or high levels.

The system demonstrated appropriate temporal stability of the MDI, which is necessary in a context where the studied measurement methods integrate measurement over a given 
duration. This temporal stability was also confirmed by the real-time monitoring (see next paragraph) for which a stability of $7 \%$ (RSD) was observed in average. This stability confirms that the measured concentration of MDI is not the result of a short, but a high concentration peak, followed by a longer period of very low concentration which, when combined, could provide a similar result over the collection period. While the longest test duration used in this study was $67 \mathrm{~min}$, the nebulizer type and the system overall configuration imply no limitations in terms of test duration. The system would therefore be fit for any recommended sampling time, e.g. from 15 min to 8 hours.

\section{Real-time monitoring of MDI using particle concentration}

Despite showing a drastic decrease in its detection efficiency for particles $<150 \mathrm{~nm}$, the AOS could be used for real-time monitoring of the concentration of MDI generated by the system. Indeed, a correlation was found between the mass concentration of particles calculated from the number concentration measurements and the MDI measured by the MAMA method. It must be noted this correlation is good only for the described conditions and parameters seen in Materials and Methods. Equation 1 presents the linear regression (origin forced to zero) describing the relationship between the two variables, where $C_{A O S}$ is the concentration of particles measured by the AOS, and $C_{M D I}$ is the concentration of MDI measured by the MAMA method. The coefficient correlation was $0.998(n=18)$.

$$
C_{A O S}=1.93 * C_{M D I}
$$

The factor of 1.9 (equation 1) between AOS and specific MDI analyses is most likely explained by the inability of AOS to measure all MDI particles and also by the AOS calibration, which had been performed with other particles with different physical properties. AOS was still a valuable and simple tool, providing the MDI concentration at any time during a generation test. The average temporal variations over the whole test were $2 \%$ and $7 \%$ for MAMA and AOS respectively, which are in the same order of magnitude.

\section{Characterization of Nebulized MDI}

The particle-size distribution, in particle number concentrations, measured by ELPI+ is presented in Figure 2. To our knowledge, this is the first time that the characterization by ELPI+ of atmospheres containing an isocyanate has been presented. Since all the air supplied to the system was HEPA filtered and the data were corrected for solvent background, it is hypothesized that the vast majority of the particles measured were MDI. Figure 2 shows that the MDI particles are in the aerodynamic diameter range of 10 to $50 \mathrm{~nm}$, which is expected, given the generation by nebulization (41). Figure 2 also shows that the distribution does not depend on the concentration, as the total aerosol fraction represented by each particle size channel does not exhibit important changes when the MDI concentration is an order of magnitude higher. 


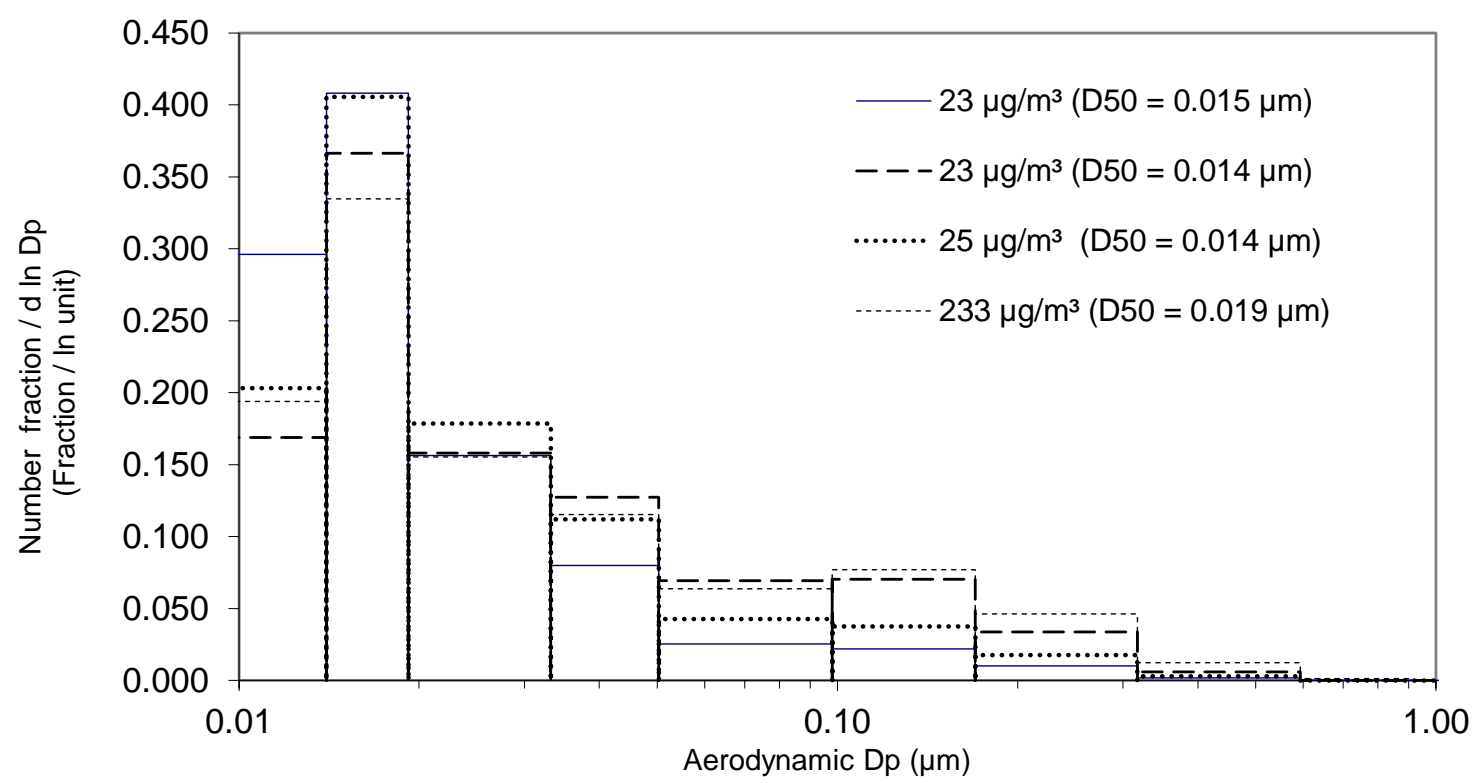

Figure 2. Particle-size distribution and median diameter (D50) (number concentration) measured by ELPI+ at four different MDI concentrations.

Since the MDI-specific analysis is a mass measurement, the ELPI+ results were expressed as a mass concentration for comparison purposes. Figure 3 shows the superimposition of the mass particle-size distributions measured by the cascade impactor (MDI specific) and the ELPI+ (nonspecific). The particle-size range of the cascade impactor from 0 to $500 \mathrm{~nm}$, illustrated by a horizontal dashed line, represents the last stage result, which is from an impregnated filter capturing both $\mathrm{MDI}$ particles and vapors. In terms of particle-size distribution, Figure 3 compares the mass measurement specific to MDI and that of non-specific particles whose particle-size separations were obtained by inertia (impaction). Though not perfect and given the very different principles of measurement involved, this agreement is significant. Indeed, MDIspecific measurements are time-integrated chemical analyses, whereas ELPI+ mass results are only calculated from real-time electrical aerosol detection and assume spherical aerosol particles. The MDI found in the stages greater than $1 \mu \mathrm{m}$ of the cascade impactor is attributed to the diffusion of MDI vapor or very fine particles. This observation was anticipated, given the nature of the substance measured, which required the use of impaction membranes impregnated with reagent, which differs from the membranes usually used in this type of device (e.g., inert particles). Since the small-particle diffusion losses are corrected in the data posttreatment algorithm, this issue does not alter ELPI+ measurements. 


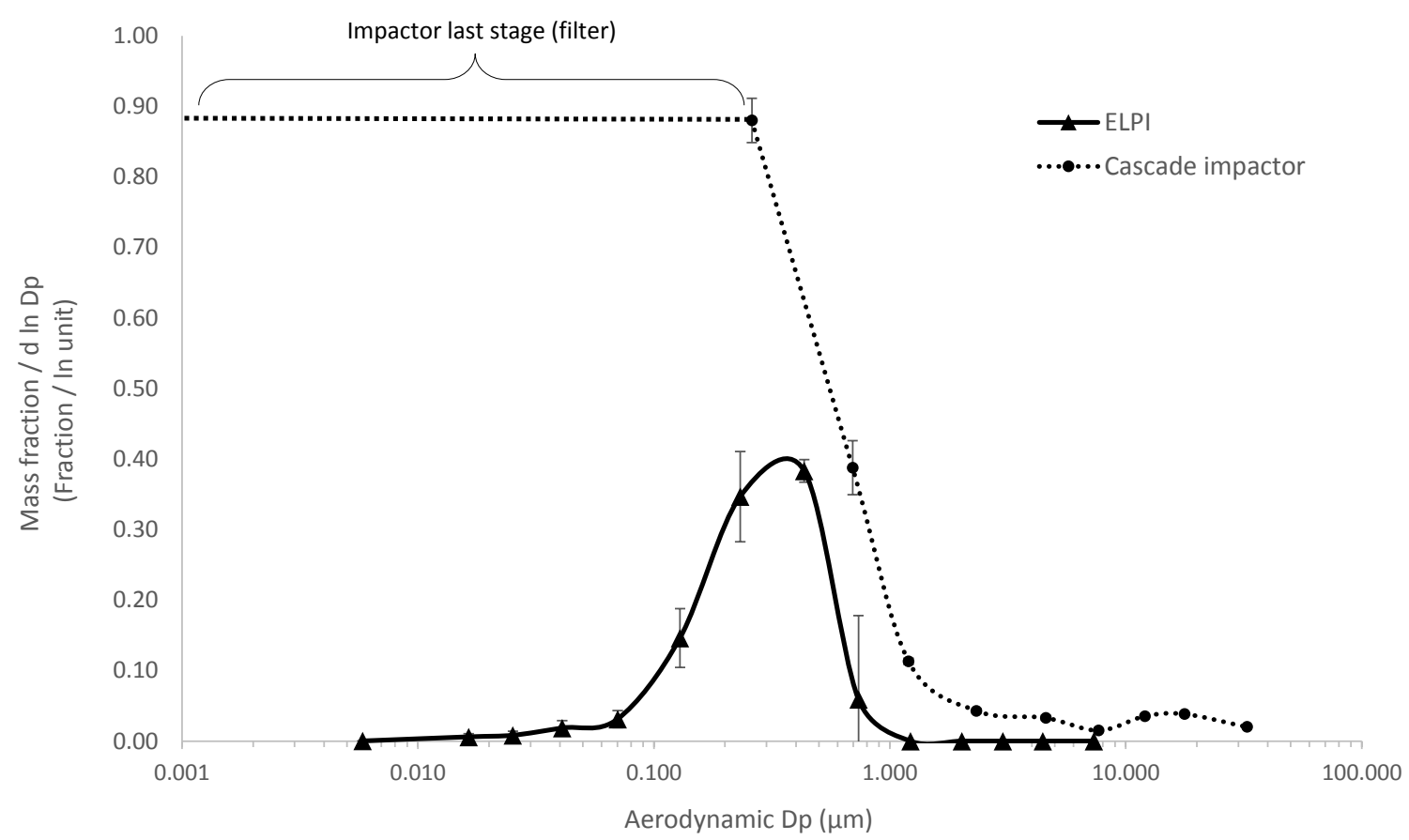

Figure 3. Particle-size distribution (mass) of MDI measured by cascade impactor $(n=2)$ and

$\mathrm{ELPI}+(\mathrm{n}=4)$.

\section{Method Comparison}

Comparison of the test results (Figure 4, tests 2 to 5 ) established that all the particles measured by the ELPI+ accounted on average for $95 \%$ and $99 \%$ of the MDI measured by the MAMA and Asset methods respectively. Assuming that a fraction of the nebulized MDI can exist in the vapor phase, these results would support the hypothesis that the majority of particles measured by ELPI+ are MDI. This also confirms the accuracy of the particle-size distribution presented in Figure 3, which shows that most of the mass of particulate MDI is found in particles of 100 to $700 \mathrm{~nm}$.

These results demonstrate the relevance of ELPI+, which provides real-time results directly comparable to the specific mass measurement of $\mathrm{MDI}$, in terms of both total concentration and particle-size distribution, provided the adjustment for particle density described in the Materials and Methods section is made. Its principle of operation, which relies on inertial impaction, demonstrates superior capacity to the aerosol particle sizer (APS) and the scanning mobility particle sizer (SMPS), which have been used in past studies on isocyanate generation systems.(26, 42) APS and SMPS are able to measure efficiently a narrower range of particle diameters ( 0.5 to $20 \mu \mathrm{m}$ for APS, and 0.01 to $1 \mu \mathrm{m}$ for SMPS). Their measurements expressed in terms of the number of particles are of limited representativeness in a context of exposure to isocyanates and, more specifically, air monitoring method characterization. In addition, using SMPS and APS coupling requires converting electrical mobility diameter to aerodynamic diameter, a conversion somewhat difficult with aerosols of complex origins. Finally, depending on APS model, counting efficiency drastically drop below $900 \mathrm{~nm}$, i.e. a particle size range of major interest as exhibited by this study (43-45).

Figure 4 also presents the MDI results for the MAMA cassette, cascade impactor, and Asset methods. There is good agreement (mean bias of 3\% with Asset set as reference) between the 
MAMA cassette and Asset methods, which are based on quite different principles of sampling and analysis. As the Asset method is a recognized reference method with well-documented performance for this type of isocyanate emission (vapor phase and fine particles), these results demonstrate the validity of the MDI results for documenting the performance of the generation system. In addition, the impactor results presented in Figure 4 (tests 2 and 3), which consist of the sum of the stages obtained to determine the particle-size distribution above (Figure 3), show very good agreement with the other two methods. This provides further confirmation of the representativeness of the particle-size distribution discussed above.

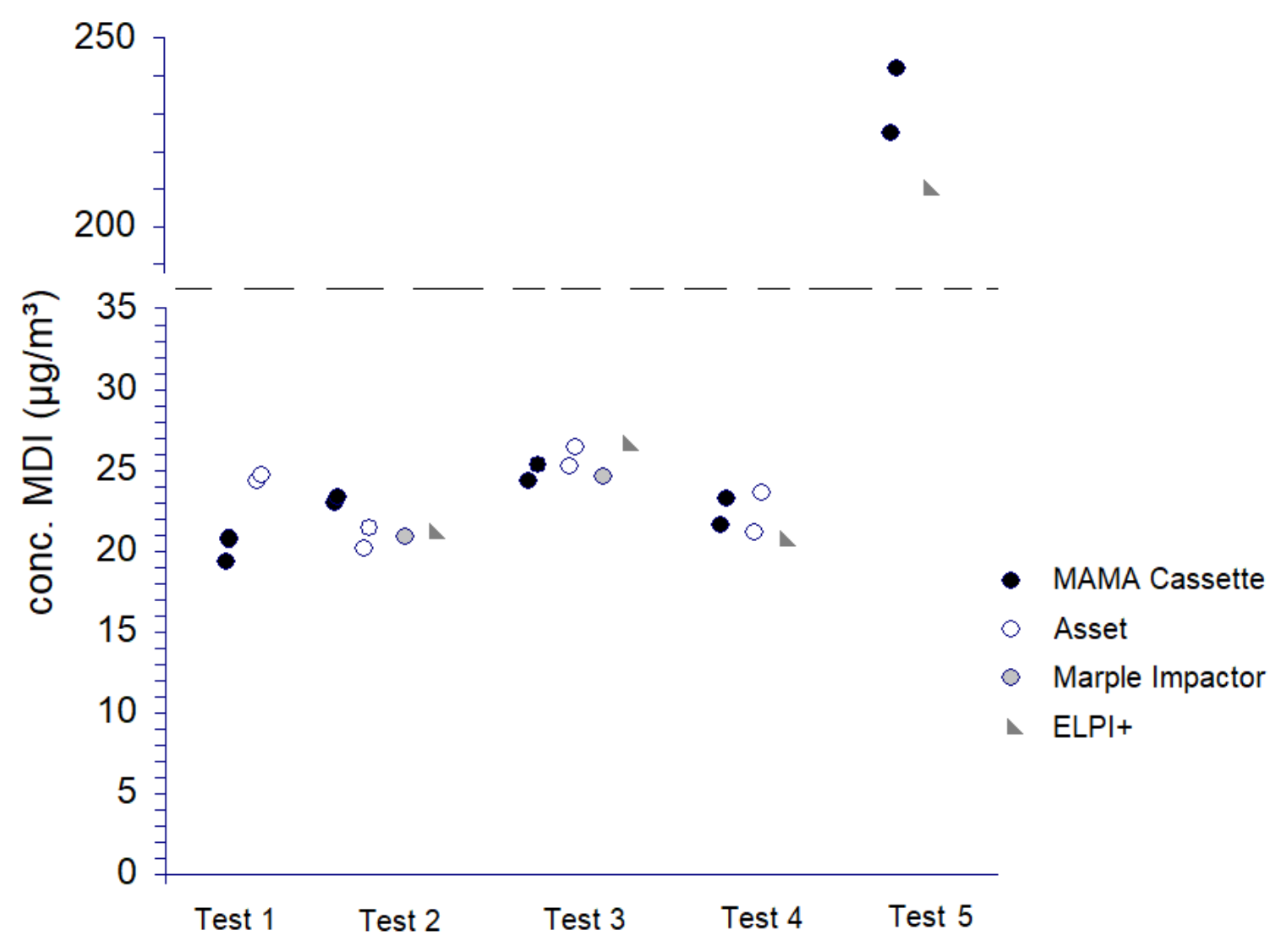

Figure 4. Five tests comparing different methods.

Vapor and Particle Phase MDI Partition

Separate analysis of the two sections of the Asset sampling device provided an estimate, according to the device's sampling principle $(26,46)$, of the physical phase partition of the MDI nebulized during the tests. Figure 5 shows that, on average, for tests 1 to 4 , the results of which are shown in Figure 4, the MDI partition was found at $8 \%$ in the vapor phase (denuder) and at $92 \%$ in the particulate phase (end filter) (standard deviation [SD] of $0.6 \%, n=8$ ). 


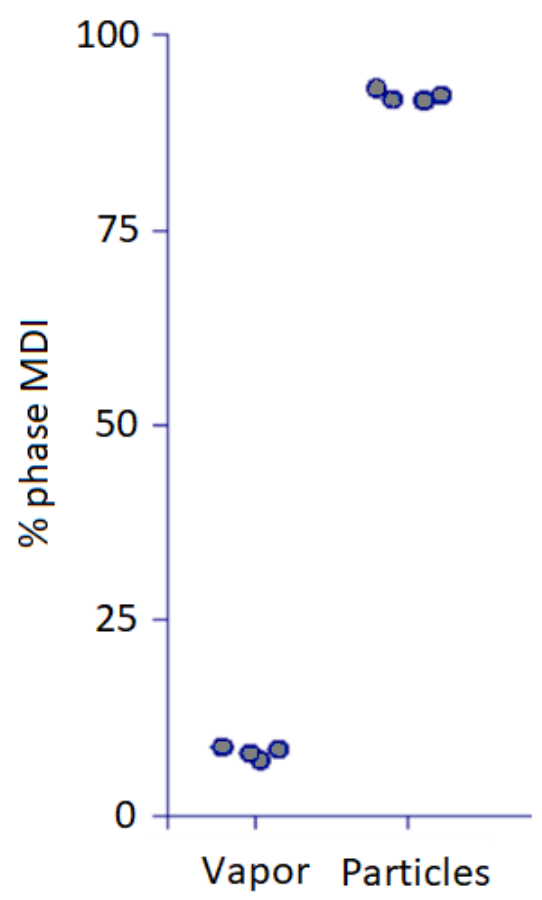

Figure 5. Trends observed in physical phase partitioning using the Asset sampler.

Some caution is called for in the interpretation of these results, as diffusion of small particles is most likely to occur in the denuder. The presence of a V-filter into the denuder tube may in fact cause a shift towards a higher proportion of vapor phase MDI than in reality. These partitioning results will be explored further in a later study.

\section{Conclusion}

A simple modular approach was developed to generate controlled MDI atmospheres (from 4 to $233 \mu \mathrm{g} / \mathrm{m}^{3}$ ) capable of reaching steady-state concentration level within 5 minutes, with welldocumented performance, such as intra- and inter-test variability (RSD of $4 \%$ and 15\%, respectively). Accuracy of the validation data was confirmed by the agreement between the routine method (glass fiber impregnated with MAMA) and the ISO 17734-1 method (Asset EZ4NCO) used as reference.

The combination of different approaches enabled an exhaustive characterization of the generated airborne MDI in terms of particle-size distribution (most of the MDI mass consisted of particles of 100 to $700 \mathrm{~nm}$ ) and physical phase partition between the vapor and particle phases ( $8 \%$ and $92 \%$ respectively), according to the Asset sampler. This study demonstrated for the first time in the scientific literature that the ELPI+ can be a highly valuable tool in providing real-time representative mass concentration levels and particle-size distribution of airborne MDI in the context of a laboratory generation system.

This in-depth validation of a modular approach, in which other components can be inserted or some replaced, makes it easier to implement the system when more complicated, more representative processes observed in occupational settings are required. Examples of these 
processes include, but are not necessarily limited to, pulverization of reactive aerosols, such as isocyanates, encountered in the application of polyurethane paints or foams.

\section{Acknowledgements}

The authors would like to thank Lucile Richard and Pierre Drouin, who helped with the laboratory analyses and the generation system setup. The authors also thank Philippe Lemay for the drawing of the system scheme. Our thanks also go to the IRSST for its financial and technical support. 


\section{References}

1. Environmental Protection Agency. Technical Overview of Volatile Organic Compounds: EPA; [Available from: https://www.epa.gov/indoor-air-quality-iaq/technical-overview-volatileorganic-compounds.

2. European Committee for Standardization. Workplace exposure - Procedures for measuring a chemical agent present as a mixture of airborne particles and vapour Requirements and test methods. EN; 2014.

3. International Organization for Standardization. (en préparation) ISO 23861 - Workplace Air - Procedures for measuring a semi-volatile chemical agent using pumped samplers Requirements and test methods ISO; 2018.

4. INRS. HAP M-332 Paris, France: INRS; 2018 [Available from: https://www.inrs.fr/publications/bdd/metropol/fiche.html?refINRS=METROPOL 332.

5. NIOSH. Polynuclear Aromatic Hydrocarbons by HPLC - 5506 Washington DC: NIOSH-CDC; 1998 [Available from: https://www.cdc.gov/niosh/docs/2003-154/pdfs/5506.pdf.

6. NIOSH. Organophosphorus Pesticides - 5600 Washington DC: NIOSH-CDC; 2016 [Available from: https://www.cdc.gov/niosh/docs/2014-151/pdfs/methods/5600.pdf.

7. Kim SW, Soderholm SC. Particle and Gas Phase Interactions in Air Sampling. Monograph of the ACGIH ${ }^{\circledR}$ Air Sampling Instruments Committee. Publication \#ASI26. Cincinnati, Ohio: American Conference of Governmental Industrial Hygienists (ACGIH); 2013.

8. American Conference on Government Industrial Hygienists. 2021 TLVs and BEls : based on the documentation of the threshold limit values for chemical substances and physical agents and biological exposure indices Cincinnati, Ohio: ACGIH; 2021. 254 p.

9. Malo JL, Ouimet G, Cartier A, Levitz D, Zeiss CR. Combined alveolitis and asthma due to hexamethylene diisocyanate (HDI), with demonstration of crossed respiratory and immunologic reactivities to diphenylmethane diisocyanate (MDI). The Journal of allergy and clinical immunology. 1983;72(4):413-9.

10. Banks DE, Butcher BT, Salvaggio JE. Isocyanate-induced respiratory disease. Annals of allergy. 1986;57(6):389-96.

11. Mapp CE, Corona PC, De Marzo N, Fabbri L. Persistent asthma due to isocyanates. A follow-up study of subjects with occupational asthma due to toluene diisocyanate (TDI). The American review of respiratory disease. 1988;137(6):1326-9.

12. Vandenplas O, Cartier A, Lesage J, Perrault G, Grammer LC, Malo JL. Occupational asthma caused by a prepolymer but not the monomer of toluene diisocyanate (TDI). The Journal of allergy and clinical immunology. 1992;89(6):1183-8.

13. Vandenplas O, Malo JL, Saetta M, Mapp CE, Fabbri LM. Occupational asthma and extrinsic alveolitis due to isocyanates: Current status and perspectives. BR J IND MED. 1993;50(3):213-28.

14. Mapp CE, Butcher BT, Fabbri LM. Polyisocyanates and their prepolymers. In: Bernstein IL, Chan-Yeung M, Bernstein DI, editors. Asthma in the Workplace. New York: Marcel Dekker, Inc; 1999. p. 457-78.

15. IFA. Institute for Occupational Safety and Health of the German Social Accident Insurance (IFA), GESTIS database: International limit values for chemical agents (Occupational exposure limits, OELs): IFA; 2021 [Available from: http://limitvalue.ifa.dguv.de/.

16. Allport DC, Gilbert DS, Outterside SM. Occupational exposure limits, stack limits and community limits. In: Sons JW, editor. MDI and TDI: safety, health and the environment: a source book and practical guide. New York2003. 
17. Streicher RP, Reh CM, Key-Schwartz R, Schlecht PC, Cassinelli ME. Chapter K Determination of Airborne Isocyanate Exposure Washington DC: NIOSH-CDC; 1998 [Available from: https://www.cdc.gov/niosh/docs/2003-154/pdfs/chapter-k.pdf.

18. Guglya EB. Determination of isocyanates in air. Journal of Analytical Chemistry. 2000;55(6):508-29.

19. Streicher RP, Reh CM, Key-Schwartz RJ, Schlecht PC, Ellen M, Paula C, et al. Determination of airborne isocyanate exposure: Considerations in method selection. American Industrial Hygiene Association Journal. 2000;61(4):544-56.

20. Ashley KE, Streicher RP, Reh CM, Key-Schwartz R, Schlecht PC, Cassinelli ME, et al. Selecting isocyanate sampling and analytical methods. Applied Occupational and Environmental Hygiene. 2002;17(3):157-62.

21. Henneken $\mathrm{H}$, Vogel M, Karst U. Determination of airborne isocyanates. Analytical and Bioanalytical Chemistry. 2007;387(1):219-36.

22. International Organization for Standardization (ISO). ISO/TR 17737 - Workplace atmospheres - Guidelines for selecting analytical methods for sampling and analysing isocyanates in air ISO; 2008.

23. Lesage J, Stanley J, Karoly WJ, Lichtenberg FW. Airborne methylene diphenyl diisocyanate (MDI) concentrations associated with the application of polyurethane spray foam in residential construction. Journal of Occupational and Environmental Hygiene. 2007;4(2):14555.

24. Karoly WJ. Stability studies of diphenylmethane diisocyanate (MDI) on glass fiber filters. American Industrial Hygiene Association Journal. 1998;59(9):645-7.

25. Bell JL. Evaluation of the OSHA 42 and NIOSH 5521 methods in determining the free isocyanate concentration in aerosols and vapor phases during application of two component 1,6-hexamethylene diisocyanate paints. Texas: Texas A\&M University; 1994.

26. Marand $\AA$, Karlsson D, Dalene M, Skarping G. Solvent-free sampling with di-n-butylamine for monitoring of isocyanates in air. Journal of Environmental Monitoring. 2005;7(4):335-43.

27. Puscasu S, Aubin S, Cloutier Y, Sarazin P, Van Tra H, Gagné S. CIP10 optimization for 4,4methylene diphenyl diisocyanate aerosol sampling and field comparison with impinger method. Annals of Occupational Hygiene. 2015;59(3):347-57.

28. Aubin S, Hamdi EM, Joly A, Sarazin P, Lesage J, Breau L, et al. On site comparison of the OSHA 42, Asset EZ4-NCO, Iso-Chek, DAN and CIP10 methods for measuring toluene diisocyanate (TDI) at a polyurethane foam factory. Journal of Occupational and Environmental Hygiene. 2020;17(5):207-19.

29. Aubin S, Hamdi EM, Joly A, Sarazin P, Lesage J, Breau L, et al. On-site comparison of the OSHA 47, Asset EZ4-NCO, Iso-Chek, DAN, and CIP10 methods for measuring methylene diphenyl diisocyanate (MDI) at an oriented-strand board (OSB) factory. Journal of Occupational and Environmental Hygiene. 2020;17(11-12):560-73.

30. Rando RJ, Poovey HG, Mokadam DR. Laboratory comparison of sampling methods for reactive isocyanate vapors and aerosols. 2002:21-37.

31. Lee CT, Poovey HG, Friedman M, Rando RJ. An HDI polyisocyanate aerosol exposure system for large-scale animal experiments. American Industrial Hygiene Association Journal. 2003;64(4):439-44.

32. Gylestam D, Gustavsson M, Karlsson D, Dalene M, Skarping G. Sampling of respirable isocyanate particles. Annals of Occupational Hygiene. 2014;58(3):340-54.

33. Lesage J, Paradis J, Obarewicz S, Ostiguy C, Karoly WJ, Van Tra H. Evaluation of the Surespot direct-reading instrument for the determination of polymeric MDI aerosols. Applied Occupational and Environmental Hygiene. 2002;17(8):543-50. 
34. International Organization for Standardization (ISO). ISO 17736 - Workplace air quality - Determination of isocyanate in air using a double-filter sampling device and analysis by high pressure liquid chromatography. ISO; 2010.

35. International Organization for Standardization (ISO). ISO 17734-1 - Workplace atmospheres - Determination of organonitrogen compounds in air uing liquid chromatography and mass spectrometry - Part 1: Isocyanates using dibutylamine derivatives. ISO; 2008.

36. Halpenny M, Brown J. ASSET ${ }^{\mathrm{TM}}$ EZ4-NCO Dry Sampler Extraction Procedure: SigmaAldrich; 2013 [Available from: https://www.sigmaaldrich.com/content/dam/sigmaaldrich/docs/Supelco/Instructions/1/ASSET EZ4-NCO Extraction.pdf.

37. Charvet A, Bau S, Bémer D, Thomas D. On the importance of density in ELPI data posttreatment. Aerosol Science and Technology. 2015;49(12):1263-70.

38. International Organization for Standardization (ISO). ISO 14382 - Workplace atmospheres - Determination of toluene diisocyanate vapours using 1-(2-pyridyl)piperazinecoated glass fibre filters and analysis by high performance liquid chromatography with ultraviolet and fluorescence detectors. ISO; 2012.

39. International Organization for Standardization. ISO 16702 - Workplace air quality Determination of total organic isocyanate groups in air using the 1-(2-methoxyphenyl)piperazine and liquid chromatography. ISO; 2007.

40. International Organization for Standardization. ISO 17735 - Workplace atmospheres Determination of total isocyanate groups in air using 1-(9-anthracenylmethyl)piperazine (MAP) reagent and liquid chromatography. ISO; 2009.

41. Li L, Zuo Z, Japuntich DA, Pui DYH. Evaluation of filter media for particle number, surface area and mass penetrations. Annals of Occupational Hygiene. 2012;56(5):581-94.

42. Dahlin J, Spanne M, Karlsson D, Dalene M, Skarping G. Size-separated sampling and analysis of isocyanates in workplace aerosols. Part I. Denuder - Cascade impactor sampler. Annals of Occupational Hygiene. 2008;52(5):361-74.

43. Peters TM, Leith D. Concentration measurement and counting efficiency of the aerodynamic particle sizer 3321. Journal of Aerosol Science. 2003;34(5):627-34.

44. Volckens J, Peters TM. Counting and particle transmission efficiency of the aerodynamic particle sizer. Journal of Aerosol Science. 2005;36(12):1400-8.

45. Armendariz AJ, Leith D. Concentration measurement and counting efficiency for the aerodynamic particle sizer 3320. Journal of Aerosol Science. 2002;33(1):133-48.

46. Forbes PBC, Rohwer ER. Chapter 5 - Denuders. In: Forbes PBC, editor. Comprehensive Analytical Chemistry. 70: Elsevier; 2015. p. 155-81. 


\title{
Electronic Supplementary Information (ESI)
}

\author{
Eight-Port Splitter Validation
}

A sodium chloride solution ( $22 \mathrm{~g}$ in $1000 \mathrm{~mL}$ of demineralized water) was nebulized into the generation system described in this study with a disposable inhalotherapy nebulizer (Glenwood Laboratories GK 1800, Canada). Several samples were collected using a pre-weight $37 \mathrm{~mm}$ polyvinylchloride membrane, porosity of $5 \mu \mathrm{m}$, installed on a cellulose pad and mounted in a $37 \mathrm{~mm}$ closed-face cassette. Air sampling was conducted at $1.5 \mathrm{~L} / \mathrm{min}$ using the same methodology described in the article (Materials and Methods). The gravimetric analysis (preweight and post-weight) was performed using a Mettler-Toledo MX-5 microbalance (Fisher Scientific, Canada) equipped with a Polonium-210 static eliminator installed in a room where humidity and temperature were controlled. Samples were kept in a desiccator for a minimum of 16 hours before the analysis. The system provided an $\mathrm{NaCl}$ concentration of between 20 and $28 \mathrm{mg} / \mathrm{m}^{3}$ from test to test. The average within-test variability ( $\mathrm{n}=4$ to 8 per test, for 3 tests), expressed as relative standard deviation, was less than $5 \%$.

Analytical Methods

\section{Chemicals}

The MDI (98\%); N-methyl-amino-methyl-9 anthracene (MAMA) (98\%); dibutylamine; DBA (> 99\%); acetone (HPLC grade), and acetic anhydride, AA, (98\%) were purchased from SigmaAldrich (USA) and were used without further purification. MDI-DBA and the MDI-DBA-d9 standard kits were supplied by Supelco (USA). MAMA-MDI was prepared by Nuchem Therapeutics (Canada). Acetonitrile (CAN), water $\left(\mathrm{H}_{2} \mathrm{O}\right)$, formic acid (FA) (optima grade), and phosphoric acid (H3PO4) (> 99\%) were obtained from Fisher Scientific (Canada). Toluene (> 99\%) was purchased from EMD Millipore (USA) and dimethylformamide from the J.T. Baker Company (Fisher Scientific, Canada). The glacial acetic and sulfuric (96\%) acids, dimethylformamide, triethylamine (HPLC grade), DMF (HPLC grade), and methanol, MeOH (HPLC grade) were obtained from J.T. Baker (Fisher Scientific, Canada).

\section{Instrumentation}

Lab preparation involved the use of an Eberbach shaker (Michigan, USA), a Branson B-52 ultrasonic bath (Danbury, USA), and a Sorvall ST 40R centrifuge (Thermo Scientific, Canada). A Zymark Turbovap LV-ZW700 evaporator was used (Biotage, USA). MAMA method samples were analyzed using an ultra-high-performance liquid chromatographic (UPLC)-photodiode array (PDA) system consisting of an LC1290 from Agilent (USA) with a Zorbax Eclipse Plus C18, $2.1 \times 50 \mathrm{~mm}, 1.8 \mu \mathrm{m}$ (Agilent, USA). The software used to operate this system and analyze the data was OpenLab CDS, also from Agilent. Asset samples were analyzed using an ultra-highperformance liquid chromatographic-mass spectrometry (UPLC-MS/MS) system consisting of a Waters Acquity UPLC coupled with a Waters Xevo TQ triple quadrupole MS (USA) equipped with an electrospray source and an autosampler having a partial loop and a needle overfill feature $(10 \mu \mathrm{L})$. The column was an Acquity UPLC BEH C18, $1.7 \mu \mathrm{m}, 2.1 \mathrm{~mm} \times 100 \mathrm{~mm}$, from Waters 
(Santry, Ireland). The software used to operate the system and analyze the data was Masslynx, V4.1, from Waters (USA).

Analysis

\section{MAMA Method}

Calibration standards with MAMA-MDI derivative were prepared in DMF to cover a concentration range of 0.015 to $0.700 \mu \mathrm{g}$ of $\mathrm{MDI}$ per $2 \mathrm{~mL}$. The mobile phase consisted of trimethylamine (TEA) buffer ( $30 \mathrm{~mL}$ TEA $+940 \mathrm{~mL} \mathrm{H2O}, \mathrm{pH} 3$ with H3PO4) (eluant A) and ACN (eluant B), respectively. Each sample was desorbed in $2 \mathrm{~mL}$ of desorption solution (60 $\mathrm{mL}$ TEA buffer $+140 \mathrm{~mL} \mathrm{ACN}$ and $400 \mathrm{~mL} \mathrm{DMF).} \mathrm{The} \mathrm{LC} \mathrm{method} \mathrm{used} \mathrm{an} \mathrm{isocratic} \mathrm{elution} \mathrm{program,} 25 \% \mathrm{~A}$ and $75 \% \mathrm{~B}$, at $1 \mathrm{~mL} / \mathrm{min}, 8 \mu \mathrm{L}$ sample injection and column kept at $30^{\circ} \mathrm{C}$. PDA detection was performed at $\lambda=254 \mathrm{~nm}$. Run time for each analysis was 8 minutes.

\section{Asset Method}

Asset standards and samples were prepared according to Halpenny et al.(1) The prepared solutions were injected $(10 \mu \mathrm{L})$ into the UPLC-MS via its autosampler, set at $15^{\circ} \mathrm{C}$. The mobile phase was composed of $\mathrm{ACN}+0.1 \% \mathrm{FA}$ (eluant $\mathrm{A}$ ) and water $+0.1 \% \mathrm{FA}$ (eluant $\mathrm{B}$ ). The run started with a gradient of $70 \%$ eluant A ( $0.5 \mathrm{~min}$.), ramped to $90 \%$ eluant A ( $2.5 \mathrm{~min}$.), held at $90 \%$ eluant $A$ ( $2 \mathrm{~min}$.), ramped to $100 \%$ eluant $A$ ( $3 \mathrm{~min}$.), and finally equilibrated at $70 \%$ eluant $A$ ( $2 \mathrm{~min}$.). The flow rate in the column was $0.6 \mathrm{~mL} / \mathrm{min}$. and the temperature was maintained at $50^{\circ} \mathrm{C}$. The Xevo TQ was used in positive mode with the capillary voltage set at $2 \mathrm{kV}$ and the source temperature at $150^{\circ} \mathrm{C}$. The desolvation temperature and flow were $500^{\circ} \mathrm{C}$ and $1,000 \mathrm{~L} / \mathrm{h}$, respectively, while the collision gas flow was set at $0.15 \mathrm{~mL} / \mathrm{min}$. The $[\mathrm{M}+\mathrm{H}]+$ for MDIDBA is $\mathrm{m} / \mathrm{z} 509.3$ and that of the internal standard, MDI-DBA-d18, is $\mathrm{m} / \mathrm{z} 527.4$. The data were acquired in multiple reaction monitoring (MRM) mode (Table S1). Manual adjustments were made on the integrations to ensure that the entire peak was covered before the data were recorded. The results were used to create a regression calibration curve with linear fit.

Table S1. MRM species calculated and measured.

\begin{tabular}{ccccc} 
Substances & $\begin{array}{c}\text { Calculated } \\
{[\mathbf{M}+\mathbf{H}]+}\end{array}$ & MRM transitions & Cone (V) & $\begin{array}{c}\text { Collision } \\
\text { energy (eV) }\end{array}$ \\
MDI-DBA & 509 & $509.3-130.2$ & 35 & 20 \\
MDI-DBA-d18 & 527 & $451.2-139.1$ & 45 & 30 \\
\hline
\end{tabular}

\section{Quality Control}

For each method, all analytical sequences incorporated quality control samples (QCS). For each sequence, the calibration standards were analyzed, followed by a reagent blank (RB), a $Q C$ spiked at the limit of quantification (QCLOQ), a QC at a value representing $50 \%$ of the dynamic range (QC50\%) and then followed by the samples. Every 10 samples, the QC50\% was analyzed to verify the calibration of the analytical run. At the end of the sequence, the RB, QCLOQ, and 
QC50\% were analyzed again, followed by the calibration standards. Each result was reported considering the fact that the QCS were in the acceptable range, which were $\pm 20 \%$ and $\pm 50 \%$ for QC50 and QCLOQ respectively.

\section{References}

1. Halpenny M, Brown J. ASSET ${ }^{\mathrm{TM}}$ EZ4-NCO Dry Sampler Extraction Procedure: SigmaAldrich; 2013 [Available from: https://www.sigmaaldrich.com/content/dam/sigmaaldrich/docs/Supelco/Instructions/1/ASSET_EZ4-NCO_Extraction.pdf. 